\title{
EL PUEBLO HUETAR Y LOS INDÍGENAS SIN PUEBLO: DIFERENCIAS Y SIMILITUDES EN CUANTO A RESIDENCIA Y OCUPACIÓN EN EL CENSO DEL 2011 DE COSTA RICA
}

\section{THE HUETAR PEOPLE AND 'TRIBELESS' INDIGENOUS PEOPLE: DIFFERENCES AND SIMILARITIES WITH REGARDS TO RESIDENCE AND OCCUPATION IN THE 2011 COSTA RICAN CENSUS}

\author{
Orlando Amaris Cervantes ${ }^{2}$ \\ o.amaris@gmail.com
}

Fecha de recepción: 21 de abril de 2016 - Fecha de aceptación: 26 de julio de 2016

\begin{abstract}
Resumen
El artículo analiza la autoidentificación, la residencia y la ocupación del pueblo huetar a partir de la información del Censo del 2011 de Costa Rica contrastando esta información con la de los "indígenas sin pueblo" y las personas autoidentificadas como "blanco o mestizo" en el Censo del 2011. Los hallazgos de esta investigación indican que, tanto por su ubicación como por sus ocupaciones, las personas autoidentificadas como huetares y como "indígenas sin pueblo" comparten características similares. Lo anterior sugiere que los individuos autoidentificados como "indigenas sin pueblo", nacidos en cantones de tradición huetar, son huetares aunque no se identifiquen como tales. Podría ser, en consecuencia, que el pueblo huetar sea entonces más numeroso de lo que expresa y permite una identificación a partir de una categoría nominal. Oportunamente, este estudio aborda también las diferencias ocupacionales que existen, en conjunto, entre las personas autoidentificadas como huetares e "indígenas sin pueblo" frente a la población "blanca o mestiza". En este sentido, se concluye que son estos últimos quienes realizan en mayor proporción las ocupaciones mejor remuneradas mientras que los huetares, por su parte, presentan también diferencias con respecto al resto de indígenas. Estas diferencias están relacionadas básicamente con la proximidad del Territorio Indígena huetar de Quitirrisí a los centros urbanos del Gran área Metropolitana (GAM) así como los lugares donde más recientemente residen los miembros de este pueblo.
\end{abstract}

Palabras clave: Identidad Indígena, Desterritorialización, Migración Indígena, Oficios Laborales, Control Cultural

\begin{abstract}
The article analyzes the residence and occupations of the Huetar people, using the 2011 Costa Rican Census; contrasting it with the residence and occupations of those who identified themselves as "tribeless indigenous" and/or "white or mixed" in this census. The findings indicate that those who self-identify as Huetar and those who do so as "Tribeless indigenous" share similar characteristics with regards to their location and their occupations; which suggests that those who identify themselves as "Tribeless indigenous", born on traditionally Huetar cantons, are in fact Huetar, even if they do not identify as such. This would mean that the Huetar people are larger than what the identification with the nominal category expresses or allows for. The study also delves into the occupational differences that
\end{abstract}

1 X Censo Nacional de Población y VI de Vivienda.

2 Investigador. Centro de investigación en Cultura y Desarrollo (CICDE) de la Universidad Estatal a Distancia (UNED). 
exist between those who self-identify as Huetar and "Tribeless indigenous", as a whole, and those who self-identify as "white or mixed"; where the latter hold the higher paying occupations, while the Huetar also show differences with regards to the other indigenous groups. These differences are fundamentally related to the Huetar territory of Quitirrisi's proximity to the Great Metropolitan Area (GAM), and to those locations where the other members of this group more recently reside.

Key Words: Indigenous Identity, Displacement, Internal Migration, Occupation, Cultural Control.

\section{Introducción}

En este artículo se parte del siguiente supuesto: cualquier grupo cultural, como en este caso el huetar, no puede ser descrito como si tuviese características permanentes en el tiempo. Lo anterior es fundamental puesto que cuando se trata de caracterizar a un pueblo indígena, es recurrente el conteo y distinción, sin criterio conocido, sobre quién es indígena y quién es mestizo. Este principio dicotómico es portado tanto por personal académico como por funcionarios estatales quienes, con argumentos racistas, ponen en duda la etnicidad de este grupo debido a que sus miembros poseen rasgos que indican, según sus impresiones, la pérdida de su cultura "original”, llegándose incluso a negar su pertenencia.

Por el contrario, desde la perspectiva que asume este documento, las formas en las que se manifiesta la cultura de un grupo están determinadas por las circunstancias externas y cambiantes a las que debieron adaptarse los actores mismos sin que esto implique necesariamente una pérdida cultural total o la pérdida de la identificación cultural grupal contrastante (Barth, 1976). Para efectos de la relación intergrupal, usualmente todos los grupos, incluidos los provenientes de la cultura dominante, hacen una selección subjetiva de los elementos que se esgrimen como distintivos frente a otros y que se destacan para diferenciar los propios de los extraños.

Si bien es cierto, desde el siglo XVI los huetares han padecido procesos de merma en el control cultural (Bonfil, 1988) resultado de la explotación y el mestizaje que provocaron que incluso su lengua desapareciera a finales del siglo XVIII (Quesada, 1999-2000), persisten rasgos identitarios y prácticas que los distinguen y permiten su autoidentificación frente a la cultura hegemónica. Muchos de estos rasgos son expuestos ampliamente en la obra de Quesada (1996; 1998), tales como el trabajo con tejidos, técnicas de cultivo, preparación de alimentos, elaboración de utensilios e instrumentos de caza y pesca, costumbres y tradición oral. No obstante, desde nuestra perspectiva, antes de hacer una enumeración de rasgos materiales o culturales -característico de una perspectiva culturalista-, más bien se destacará en esta oportunidad los procesos de autoidentificación cultural colectiva, por medio de la cual los miembros de un grupo se adscriben al mismo reconociéndose como parte de una tradición cultural a la que llaman pueblo huetar, en donde es este legado el que les diferencia de otros grupos.

Lo anterior es también sustentado teóricamente por Barth (1976) y jurídicamente por el Convenio 169 de la Organización Internacional del Trabajo (OIT) suscrito por Costa Rica en 1993. En el primer caso, siguiendo la perspectiva Barth (1976), es posible afirmar que existe una comunidad étnica huetar ya que, entre otros factores, sus miembros se identifican a sí mismos como tales y son así identificados por los miembros de otros grupos. En segundo lugar, relacionado con el Convenio 169 de la OIT (2008), se considera indígena a un pueblo si es descendiente de quienes habitaban en el país o la región al momento de la conquista, colonización o antes del establecimiento de las fronteras estatales. Sumado a lo anterior, según el primer artículo del Convenio, un criterio fundamental es la conciencia que sobre su propia identidad indígena pueda tener dicho grupo además de las diferencias sociales, culturales y económicas que logren distinguirlo de otros sectores de la colectividad nacional. 


\section{Los lugares de residencia del pueblo indígena huetar según el Censo del 2011 y antecedentes}

En el Censo del 2011, realizado en Costa Rica por el Instituto Nacional de Estadística y Censos (INEC), se registran 3461 personas que se autoidentificaron como indígenas pertenecientes al pueblo huetar en todo el territorio nacional (INEC, 2011). La anterior cantidad representa el 5\% de los 69629 indígenas miembros de alguno de los ocho pueblos originarios que hay en Costa Rica (Figura 1). Según esta misma fuente, los huetares están presentes en todas las provincias del país, especialmente en la provincia de San José (Figura 2); allí habitan 2347 miembros de este pueblo (68\%). Le siguen las provincias de Alajuela y Limón, las cuales albergan a 296 y 228 personas huetares, respectivamente (INEC, 2011). Es también importante indicar que en la provincia de San José se encuentran los dos territorios indígenas de este pueblo oficialmente declarados por el Estado costarricense: Quitirrisí y Zapatón ${ }^{3}$. Estos territorios cubren una superficie de 5515 ha. El primero de ellos, establecido en 1976, se ubica en el cantón de Mora ${ }^{4}$ y tiene una extensión de 2660 ha. El segundo, Zapatón, creado posteriormente, en 1982, se localiza al sur del cantón de Puriscal y cuenta con 2855 ha (Borge, 2007). Según el INEC (2011), en ambos territorios, habitan 1354 indígenas huetares, es decir, el 39\% de la población total de huetares del país.

Figura 1

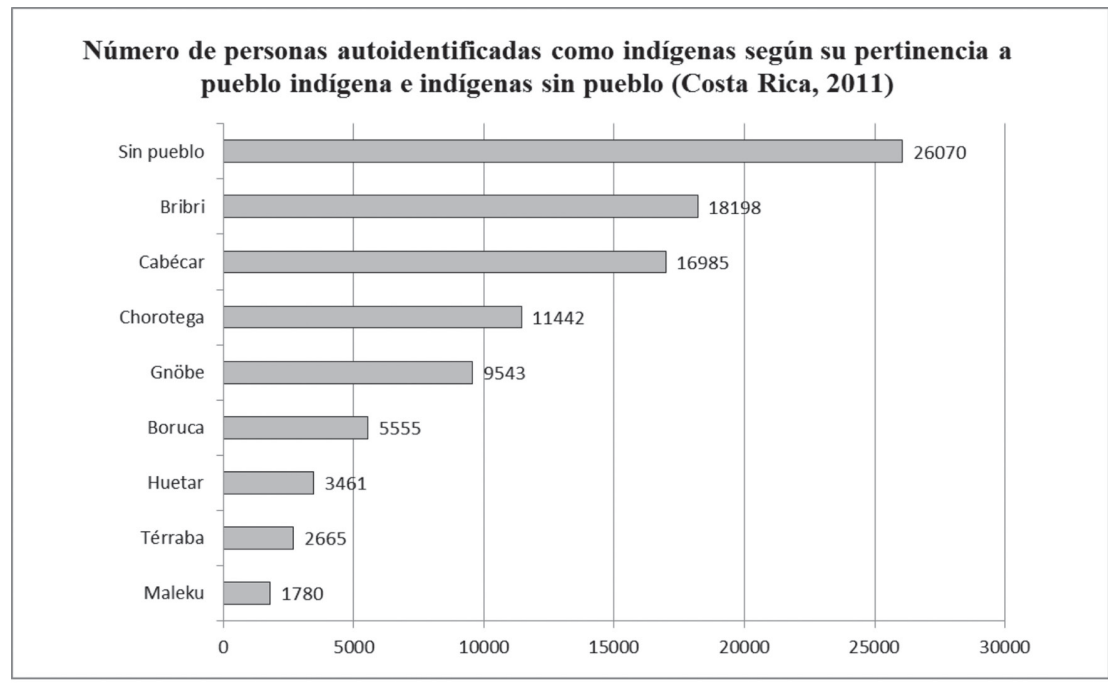

Fuente: Elaboración propia a partir de INEC (2011)

3 El Territorio Indígena de Quitirrisí fue establecido en 1976 mediante el Decreto No. 6036-G, publicado en La Gaceta 113 del 12 de junio de 1976; otros decretos posteriores relacionados son el Decreto No. 10707-G, publicado en La Gaceta 210 del 8 de noviembre de 1979; Decreto No. 29452-G, publicado en La Gaceta 93 del 16 de mayo del 2001. El Territorio Indígena de Zapatón fue establecido en 1981 mediante decreto No. 12812-G, publicado en La Gaceta 151 del 10 de agosto de 1981; otro decreto relacionado es el No. 29453-G, publicado en La Gaceta 93 del 16 de mayo del 2001.

4 En este artículo se parte de que a partir modificaciones implicadas en el Decreto No. 29452-G, publicado en La Gaceta 93 del 16 de mayo del 2001 se reforma el Territorio Indígena de Quitirrisí cubriendo a partir de ese momento 2660 ha. Lo anterior es sustentado por fuentes posteriores a dicho decreto como Borge (2007) en un informe del Programa de Regularización del Catastro y Registro. 
Figura 2

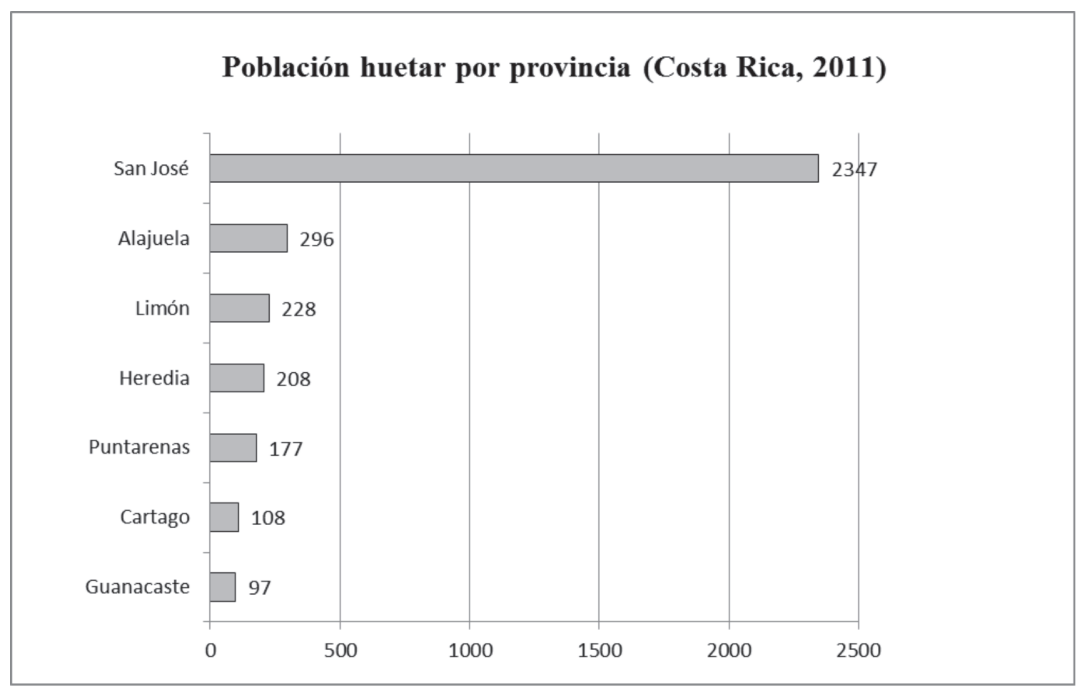

Fuente: Elaboración propia a partir de INEC (2011)

Por otra parte, muchos de los desafíos que enfrenta el pueblo huetar son comunes a los que también pesan sobre otros pueblos originarios en el país siendo denunciados por ellos mismos y además referidos en recientes informes de la Defensoría de los Habitantes (2010 y 2014) y en el informe de la Oficina del Alto Comisionado de las Naciones Unidas para los Derechos Humanos (ONU, 2011).

Entre las diferentes problemáticas ante las cuales intentan resistir estos pueblos se destaca la tenencia de tierra de buena y mala $\mathrm{fe}^{5}$ entre personas no indígenas dentro de los Territorios quienes reiteradamente han usurpado la tierra y los recursos presentes en estos en detrimento de la gestión que los pueblos originarios puedan ejercer; situación que ha generado en los indígenas el abandono de sus territorios legalmente instituidos y su desplazamiento hacia otras regiones en busca de fuentes laborales pese a que se cuenta con legislación nacional pertinente desde 1977 que en principio faculta al Estado a reducir la presencia de no indígenas dentro de los Territorios Indígenas.

Es oportuno señalar que por ley las tierras dentro de los territorios han sido declaradas como "inalienables, imprescriptibles, no transferibles y exclusivas para las comunidades indígenas que las habitan [...]" (Art.3. Ley Indígena de 1977). En la Figura 3 se observa que en los territorios huetares $44 \%$ de sus habitantes son no indígenas; el pueblo maleku, cabe decir, tiene la peor de las condiciones en este aspecto, ya que en su territorio (Guatuso) los no indígenas llegan a ser el 65\% de la población allí residente (INEC, 2011). En el caso de los huetares, la preocupante disparidad en el acceso a la tierra entre indígenas y mestizos en Quitirrisí y Zapatón bien podría ser la causa principal de que solo el 14\% de la población indígena en Quitirrisí se dedique a labores agropecuarias mientras que en Zapatón sea el 58\% de su población (INEC, 2011a).

5 Los propietarios de buena fe son aquellos propietarios no indígenas que tenían sus propiedades a derecho y vivían dentro de los territorios indígenas al momento del establecimiento del Territorio Indígena. Por lo tanto su presencia es legal y la única forma de desalojarlos es por medio de la compra de los terrenos por el Estado. Por otra parte, se tiene a los propietarios no indígenas de mala fe, quienes han adquirido tierras después de la creación del Territorio y por tanto su presencia es ilegal. 
Figura 3

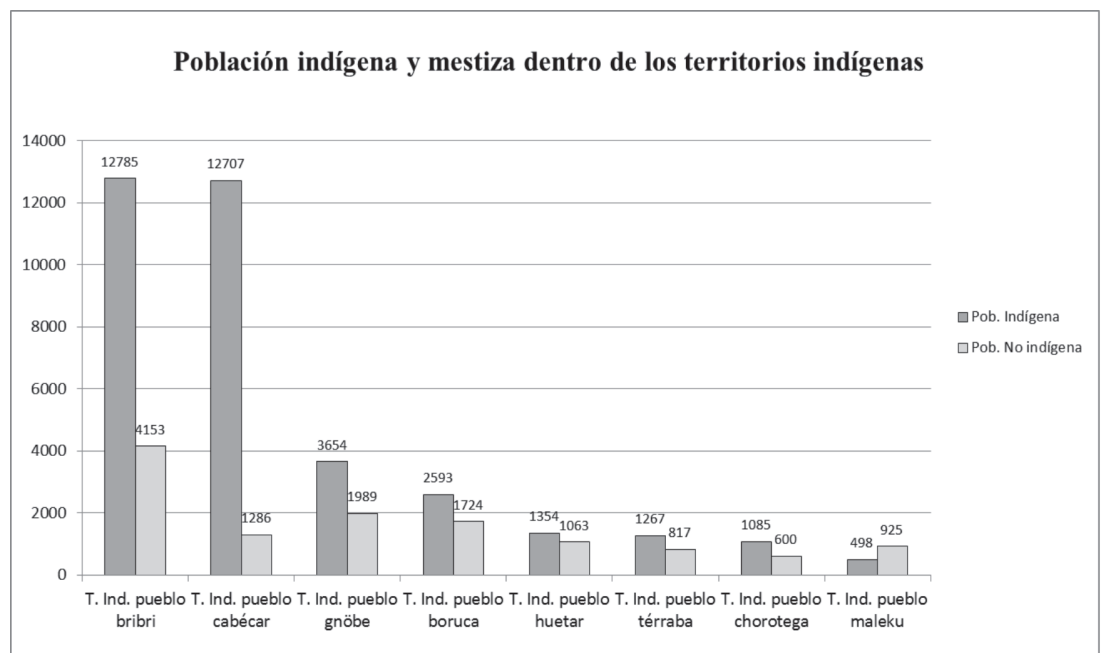

Fuente: Elaboración propia a partir de INEC (2011).

En sus dos territorios, Quitirrisí (cantón de Mora), con 2660 ha., y Zapatón (cantón de Puriscal), con 2855 ha., los huetares apenas ocupan respectivamente 9\% (239 ha.) y 20\% (571 ha.) de la tierra frente a una población no indígena creciente (Borge, 2007). Según cálculos del autor de este artículo, basados en Borge (2007) e INEC (2011a), en promedio en Quitirrisí le corresponden 0,24 ha. para cada indígena frente a 2,5 ha. para cada no indígena. Más grave todavía en Zapatón porque en promedio le corresponde 1,61 ha. a cada indígena frente a 23,5 ha. a cada no indígena. Estas cifras, por supuesto, no consideran las desigualdades que puedan existir en cuanto a la tenencia de la tierra tanto a lo interno de los indígenas como de los mestizos. Además, para el caso de Quitirrisí, la falta de tierra donde practicar la agricultura, la carencia de incentivos para desarrollarla, y su cercanía a las principales ciudades del Valle Central hace que progresivamente miembros de este grupo se desplacen a estos centros urbanos a trabajar principalmente en actividades mal remuneradas.

Los datos anteriores contrastan con la información que se dispone sobre el territorio histórico huetar anterior a la llegada de los europeos en el siglo XVI. Sobre este punto en particular, si se toma como referencia los estudios lingüísticos de Quesada $(1996,1998)$ y el empleo de herramientas propias de los Sistemas de Información Geográfica (SIG), se concluye que el territorio histórico huetar cubría más de 964.600 ha (Amaris, 2015). Se hace evidente que en la actualidad los territorios huetares suman apenas $0,6 \%$ de lo que fue su territorio histórico precolombino, pues antes del contacto, la extensión de este último, comprendía la totalidad del Valle Central, cubriendo incluso hasta la parte central de lo que hoy es la provincia de Puntarenas, la cordillera de Tilarán y Turrialba.

\section{Los indígenas sin pueblo y su presencia en cantones de tradición huetar}

En vista de estas condiciones, tan cambiantes como expulsivas, algunas de ellas descritas por Amaris (2015), y que implicaron a su vez flujos migratorios desde el cantón de Mora en la primera mitad del siglo XX, es pertinente la construcción de un referente antropológico sobre la validez de la autoidentificación como indígenas en general y como huetares en particular, con el fin de profundizar 
en la información disponible en la base de datos electrónica REDATAM del INEC, producto del censo del 2011 y relacionada con la ubicación, el lugar de nacimiento, y rasgos socioeconómicos generales tanto de este pueblo como de los indígenas sin pueblo. Estos últimos, según el INEC (2011), son personas que se autoidentifican como indígenas pero que no se reconocen como parte de algún pueblo entre los enumerados en la boleta censal lo cual, desde nuestra perspectiva, no les excluye sentirse parte de un legado precolombino o bien un mecanismo de diferenciación frente a la población "blanca o mestiza"6. En este sentido, el ejercicio que se propone en este artículo concentra también su atención sobre los indígenas sin pueblo en vista de que se trata de una población que se clasifica a sí misma como indígena habitando en cantones donde al menos para los siglos XIX y XX se presenta una predominancia huetar. La tesis de Quesada (1996) indica que, aparte de la presencia de huetares en Mora y Puriscal, también se encuentran familias de este pueblo en Acosta, y especialmente en los cantones de destino de las migraciones, señaladas también en Amaris (2015), como Parrita y Quepos, entre otros cantones del Pacífico Central.

En la Tabla 1, se destacan dentro de la provincia de San José los cuatro cantones con mayor presencia de personas que se autoconsideran indígenas huetares en el 2011: Mora, Puriscal, San José y Acosta. En el cantón de Mora, como ya se ha dicho, se encuentra el Territorio Indígena de Quitirrisí, en los distritos de Colón y Tabarcia. Al revisar la Tabla 2, según INEC (2011) en este cantón residen 1159 huetares de los cuales 1105 son nacidos en este cantón. Además, el censo en cuestión nos informa también que 999 huetares habitan el Territorio Indígena de Quitirrisí. En otras palabras, fuera del territorio indígena, pero dentro del cantón de Mora viven 160 indígenas quienes representan el 14\% del total de la población de huetares de ese cantón. Los indígenas huetares que viven en Mora representan el 4,4\% de los 26294 habitantes totales de este cantón.

6 En la boleta censal del 2011 las categorías de autoidentificación blanco y mestizo se usaron como sinónimos en una misma categoría "Blanco o mestizo" mientras que, por ejemplo, negro y mulato sí estaban adecuadamente separados permitiendo a quienes se autoidentificaran como tales escoger uno u otro y, por tanto, lograr una mayor especificidad en la respuesta. De esta manera, al igualar blanco y mestizo en la categoría "blanco o mestizo" tal como el INEC la entiende, se impidió a quienes se autoidentificaran como mestizos, por ejemplo, separarse de la categoría blanco y además los adicionó a este último grupo reforzándose así la idea, desde nuestra perspectiva, de una mayoría supuestamente blanca en Costa Rica. 
Tabla 1

Indígenas huetares de la provincia de San José, por cantón, según lugar de nacimiento (2011)

\begin{tabular}{|l|r|r|r|r|}
\multicolumn{1}{c|}{ Cantón } & Nacidos en es te cantón & Nacidos en otro cantón & \multicolumn{1}{c|}{ Total } \\
\hline San José & 94 & 57 & 151 \\
\hline Escazú & 14 & 5 & 19 \\
\hline Desamparados & 13 & 42 & 55 \\
\hline Puriscal & 461 & 88 & 549 \\
\hline Tarrazú & & 1 & 1 \\
\hline Aserrí & 4 & 7 & 11 \\
\hline Mora & 1105 & 54 & 1159 \\
\hline Goicoechea & 20 & 35 & 55 \\
\hline Santa Ana & 20 & 15 & 35 \\
\hline Alajuelita & 25 & 10 & 35 \\
\hline Vásquez de Coronado & 9 & 21 & 30 \\
\hline Acosta & 43 & 6 & 49 \\
\hline Tibás & 11 & 24 & 35 \\
\hline Moravia & 8 & 15 & 23 \\
\hline Montes de Oca & 5 & 25 & 30 \\
\hline Turrubares & 5 & 12 & 17 \\
\hline Curridabat & 10 & 15 & 25 \\
\hline PérezZeledón & 15 & 11 & 26 \\
\hline León Cortés Castro & 3 & 1 & 4 \\
\hline Total & 1865 & 444 & 2309 \\
\hline & & &
\end{tabular}

Fuente: Elaboración propia con datos del INEC (2011)

Por distrito se encuentra que, en el cantón Mora (Tabla 2), 280 huetares habitan en el distrito de Colón siendo 260 nacidos en este mismo cantón; 352 residen en el distrito de Guayabo siendo 331 nacidos en este cantón; 527 viven en el distrito de Tabarcia de los cuales 514 nacieron en este cantón; mientras que en Piedras Negras y Picagres apenas se censaron respectivamente dos y siete indígenas sin pueblo pero nacidos en Mora, como se muestra a continuación.

Tabla 2

Indígenas huetares y sin pueblo del cantón de Mora, por distrito, según lugar de nacimiento (2011)

\begin{tabular}{|l|r|r|r|r|}
\hline \multicolumn{1}{|c|}{ Dis tritos } & Nacidos en Mora & Nacidos en otro cantón & Sin Pueblo nacidos en Mora & Sin Pueblo nacidos en otro cantón \\
\hline Colón & 260 & 20 & 70 & 32 \\
\hline Guayabo & 331 & 21 & 11 & 11 \\
\hline Tabarcia & 514 & 13 & 59 & 13 \\
\hline Piedras Negras & & & 2 & 7 \\
\hline Picagres & & & 149 & 56 \\
\hline Total & 1105 & 54 & & \\
\hline
\end{tabular}

Fuente: Elaboración propia con datos del INEC (2011)

Por su parte, en el cantón de Puriscal, como se indica en la Tabla 3, se registran 461 indígenas huetares nacidos en ese cantón y 88 huetares que nacieron en un cantón diferente (INEC, 2011). Es oportuno 
señalar que los 549 indígenas huetares que habitan Puriscal representan el 1,7\% de los 33004 habitantes que tiene en total dicho cantón. Al sur del cantón de Puriscal se encuentra el distrito de Chires y allí se ubica el Territorio Indígena de Zapatón dentro del cual viven 355 indígenas huetares. Sin embargo, el cantón de Puriscal tiene 549 huetares. Dicho de otra manera, los huetares fuera del Territorio Indígena de Zapatón pero dentro del cantón de Puriscal suman 194 individuos (lo que significa el 35\% de la población de huetares del cantón de Puriscal). Nótese en la Tabla 3 que en su mayoría viven en los distritos de Santiago, Mercedes Sur, San Antonio y Barbacoas. Llama la atención, además, que es en estos distritos donde también se concentra una importante población que se considera a sí misma como indígena sin pueblo.

Tabla 3

Indígenas huetares y sin pueblo del cantón de Puriscal, por distrito, según lugar de nacimiento (2011)

\begin{tabular}{|c|c|c|c|c|}
\hline Distritos & Nacidos en Puriscal & Nacidos en otro cantón & Sin Pueblo nacido en Puris cal & Sin pueblo nacidos en otro cantón \\
\hline Santiago & 42 & 10 & 106 & 39 \\
\hline Mercedes Sur & 27 & 4 & 34 & 8 \\
\hline Barbacoas & 11 & 2 & 74 & 28 \\
\hline Grifo Alto & 1 & & 4 & \\
\hline San Rafael & 2 & & 4 & \\
\hline Candelarita & & & 27 & 10 \\
\hline Desamparaditos & 2 & & 1 & \\
\hline San Antonio & 14 & 7 & 28 & 13 \\
\hline Chires & 362 & 65 & 14 & 2 \\
\hline Total & 461 & 88 & 292 & 100 \\
\hline
\end{tabular}

Fuente: Elaboración propia con datos del INEC (2011).

En cuanto al cantón de Acosta, mencionado por Bozzoli (1973. Citado en Quesada, 1996), en la Tabla 4 se anota que de los 49 individuos que se autodefinieron como huetares, 43 de ellos son nacidos en ese cantón. Al aproximarse a la ubicación distrital de estas personas se encuentra que 32 de ellas habitan en el distrito de Sabanillas y nueve en el distrito de Palmichal. Es de subrayar que en el distrito de San Ignacio de este cantón, 114 personas se hayan autoidentificado como indígenas sin pueblo, de las cuales 92 afirmaron haber nacido en ese cantón. En el distrito de Sabanillas, Guatil y Palmichal, 21, 20 y 10 personas, respectivamente, se identificaron como indígenas sin pueblo y sostienen haber nacido allí (INEC, 2011). Los indígenas sin pueblo en el cantón de Acosta suman 182 personas de las cuales 143 sostienen haber nacido en ese cantón.

Tabla 4

Indígenas huetares y sin pueblo del cantón de Acosta, por distrito, según lugar de nacimiento (2011)

\begin{tabular}{|l|r|r|r|r}
\hline Dis tritos & Nacidos en Acosta & Nacidos en otro cantón Sin pueblo nacidos en Acos ta & Sin pueblo nacidos en otro cantón \\
\hline San Ignacio & 2 & & 92 & 22 \\
\hline Guaitil & & 6 & 20 & 3 \\
\hline Palmichal & 9 & & 10 & 10 \\
\hline Cangrejal * & 32 & & 21 & 4 \\
\hline Sabanillas & 43 & 6 & 143 & 39 \\
\hline Total & & & 4 \\
\hline
\end{tabular}

Fuente: Elaboración propia con datos del INEC (2011)

A manera de síntesis, en los tres cantones de la provincia de San José, Mora, Puriscal y Acosta, podemos destacar tanto una concentración de la población huetar como la existencia de una población que se considera a sí misma como indígena sin pueblo. Al respecto, es pertinente mencionar el mayor peso relativo de la población huetar con respecto a otras poblaciones de otros pueblos indígenas e indígenas sin pueblo en Mora y Puriscal, pero no en Acosta (86\% de la población indígena en Mora; 58\% de la 
población indígena en Puriscal; 20\% de la población indígena en Acosta). Vemos cómo, en las Figuras 4,5 y 6 , que se presentan a continuación, la población que dice ser indígena sin pueblo representa un $12 \%$ (Mora), 36\% (Puriscal) y 67\% (Acosta) de la población total que se considera indígena en esos cantones y que nacieron allí. Para el caso del cantón de Mora es importante destacar la presencia de indígenas sin pueblo nacidos en ese cantón en los distritos próximos colindantes con el Territorio indígena de Quitirrisí como lo son Colón y Tabarcia.

Adicionalmente, es oportuno señalar que la mayoría representada en $44 \%$ de los indígenas sin pueblo nacidos en Mora se encuentran en el distrito de Colón mientras que el 40\% se ubica en Tabarcia. Dicho en otras palabras $88 \%$ de los indígenas sin pueblo nacidos en este cantón habitan precisamente en los dos cantones que albergan al Territorio Indígena de Quitirrisí. Es necesario a su vez recordar que en el distrito de Colón se ubica Ciudad Colón, principal centro urbano del cantón, mientras que en el distrito de Tabarcia se encuentra otro importante poblado con el mismo nombre, lo que podría ocultar que buena parte de los indígenas sin pueblo en esos distritos vivan en estos poblados y no en las partes más próximas y rurales de estos distritos allende al Territorio Indígena de Quitirrisí.

Para el caso de Puriscal es importante indicar que el 36\% de la población autoidentificada como indígena sin pueblo en ese cantón se encuentra en el distrito de Santiago donde se halla, justamente, la cabecera municipal y el mayor centro de comercio. En segundo lugar, se encuentra que $25 \%$ de los indígenas sin pueblo se sitúan en el distrito de Barbacoas, muy próximo a dicha cabecera. Finalmente, en el distrito de Chires, donde se encuentra el Territorio Indígena de Zapatón, habita apenas el 5\% de los indígenas sin pueblo de este cantón.

Colindante, en el cantón de Acosta, en el distrito de San Ignacio habita el 64\% de la población indígena sin pueblo nacida en dicho cantón. Es decir, el distrito donde se localiza el centro poblacional más grande. Obsérvese que, en el distrito de Palmichal, próximo al Territorio Indígena de Quitirrisí, pero con menor población que Tabarcia de Mora, solo viven 7\% de los indígenas sin pueblo nacidos en Acosta.

Si bien no se puede concluir, contundentemente, que las personas indígenas sin pueblo sean indígenas huetares, por lo menos para el caso de los cantones de Mora, Puriscal y Acosta, la información histórica y la fuerte presencia huetar en la zona, ya señalada en investigaciones anteriores, así lo sugieren. En el caso de Acosta, es llamativa la gran cantidad de población que se reconoce como indígena pero que no logra identificarse en algún pueblo. En todo caso, el considerarse a sí mismo indígena es una manera de distinguirse frente a los "blancos o mestizos" o, expresado de otra forma, en oposición a los que no son indígenas, asunto relevante a destacar en el presente análisis.

Figura 4

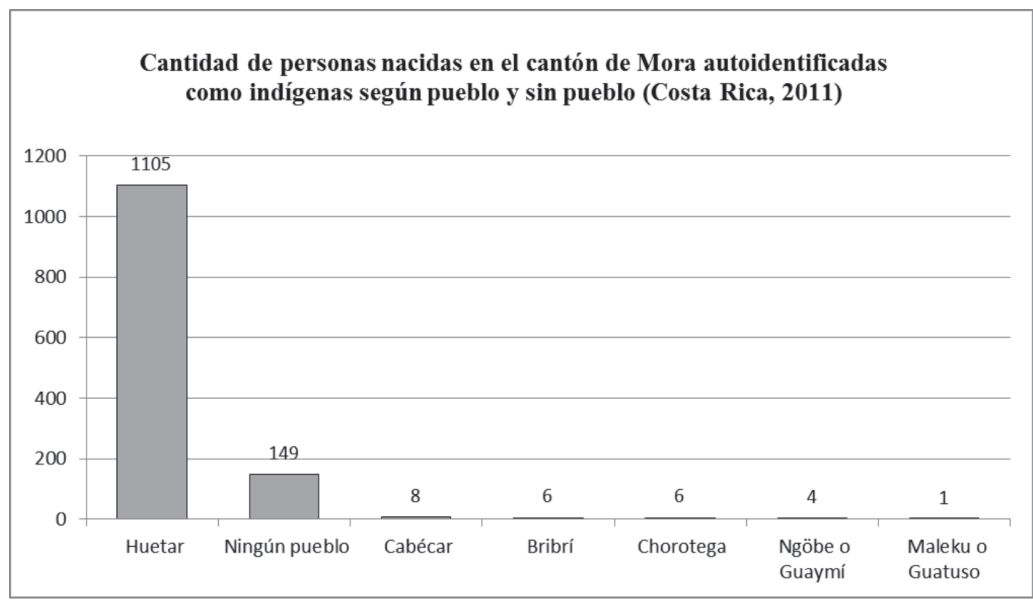

Fuente: Elaboración propia con datos del INEC (2011) 
Figura 5

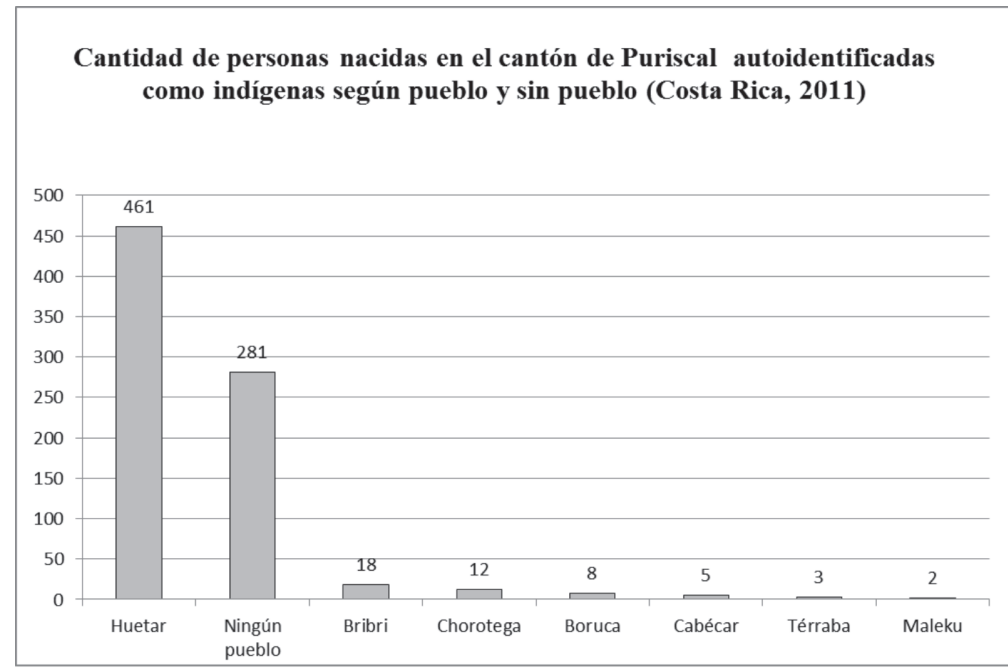

Fuente: Elaboración propia con datos del INEC (2011)

Figura 6

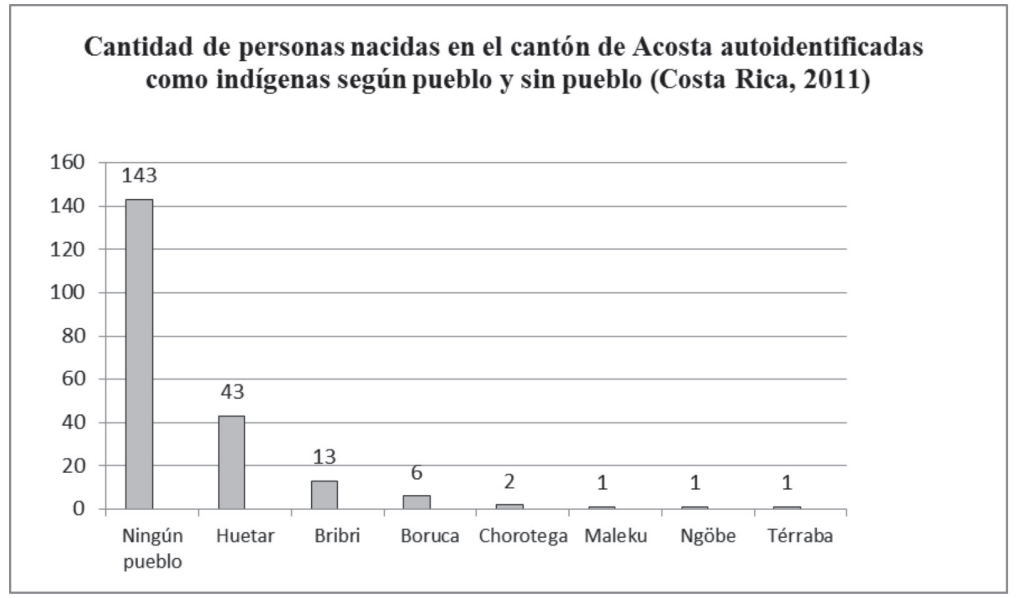

Fuente: Elaboración propia con datos del INEC (2011)

Para el caso del cantón de San José, en la provincia de San José, el censo del 2011 muestra una diversidad mayor de personas que se reconocen de distintos pueblos indígenas diferentes al huetar. $\mathrm{Al}$ respecto, encontramos una mayor presencia de indígenas bribris, borucas y chorotegas que en los demás cantones. Esta situación es entendible al ser un importante centro urbano y polo de atracción para migrantes laborales. En este caso, es difícil realizar una relación entre los indígenas sin pueblo y del pueblo huetar en particular y por ello se ha desestimado en esta oportunidad.

Por otro lado, también es importante observar la presencia de miembros del pueblo huetar en los cantones del Pacífico Central, a saber San Mateo y Orotina (Provincia de Alajuela), Puntarenas, Esparza, Montes de Oro, Garavito y Parrita (Provincia de Puntarenas) dado que toda esta zona se abrió a la colonización agrícola, facilitado con la apertura del tren al Pacífico (Bartels y Martínez, 2008; 
2010. Botey, 1999. Amaris, 2015) y, en consecuencia, se convirtió en un polo de atracción tanto para la población indígena huetar como para los mestizos empobrecidos, al contar ésta con tierra disponible y suelos aptos para iniciar el cultivo de productos de subsistencia entretanto la parte central del país se concentraba en el café como cultivo de exportación y factor cambiante de su paisaje (Amaris, 2015).

En el caso de los cantones alajuelenses de San Mateo y Orotina, en su conjunto tienen apenas cuatro huetares que afirman haber nacido en estos cantones, mientras que otros seis que residen allí nacieron en otros cantones (Tabla 5). En cuanto a las personas que se consideran indígenas y no se adscribieron a ningún pueblo, estos dos cantones tampoco tienen la relevancia que tienen otros en la provincia de Alajuela. En este sentido, 33 personas nacidas en Orotina se consideran indígenas sin pueblo mientras que en San Mateo solo 11. Por otra parte, 44 indígenas sin pueblo residen en Orotina habiendo nacido en otros cantones mientras que en San Mateo solo diez. Sin embargo, notamos cómo en los cantones alajuelenses de San Carlos, Alajuela y San Ramón hay más personas que se identifican como huetares que en los cantones que se incluyen en la región Pacífico Central (San Mateo y Orotina). Una posible explicación es que en las últimas décadas se hayan dado flujos migratorios de huetares desde cantones periféricos de la provincia de Alajuela hacia aquellos con centros urbanos alajuelenses más importantes y con mayores fuentes de empleo asemejándose de algún modo, por ejemplo, a la relación existente entre Quitirrisí y San José. Considerando lo anterior es necesario también preguntarse sobre los posibles tránsitos de la población huetar que se han dado a los 50 años siguientes al período en estudio abarcado por Amaris (2015) así como el nacimiento de hijos e hijas de miembros de este pueblo.

Tabla 5

Indígenas huetares y sin pueblo, residentes en la provincia de Alajuela, según y lugar de nacimiento (2011)

\begin{tabular}{|c|c|c|c|c|}
\hline Cantones & Nacidos en este cantón & Nacidos en otro cantón & Sin pueblo nacidos en este cantón & Sin pueblo nacidos en otro cantón \\
\hline Alajuela & 39 & 57 & 663 & 606 \\
\hline San Ramón & 21 & 16 & 159 & 164 \\
\hline Grecia & 4 & 9 & 137 & 110 \\
\hline San Mateo & 1 & 3 & 11 & 10 \\
\hline Atenas & 2 & 2 & 67 & 28 \\
\hline Naranjo & 5 & 7 & 77 & 57 \\
\hline Palmares & 10 & 3 & 97 & 64 \\
\hline Poás & 3 & 6 & 37 & 33 \\
\hline Orotina & 3 & 3 & 33 & 44 \\
\hline San Carlos & 49 & 19 & 465 & 294 \\
\hline Zarcero & 1 & & 25 & 6 \\
\hline Valverde Vega & 4 & 5 & 50 & 25 \\
\hline Upala & 13 & 3 & 110 & 32 \\
\hline Los Chiles & 2 & & 55 & 29 \\
\hline Guatuso & & 1 & 11 & 6 \\
\hline Total & 157 & 134 & 1997 & 1508 \\
\hline
\end{tabular}

Fuente: Elaboración propia con datos del INEC (2011)

En cuanto a los cantones puntarenenses que también forman parte del Pacífico Central (Puntarenas, Esparza, Montes de Oro, Garavito, Parrita y Aguirre), según la Tabla 6, las principales concentraciones de indígenas huetares e indígenas sin pueblo se da en los cantones de Puntarenas, Aguirre -donde Quesada (1996) ubicó huetares en San Gerardo, Cerritos y El Nene- y Parrita. En el caso del cantón de Puntarenas, de 48 huetares registrados allí, 12 habitan en los distritos de El Roble y 10 en el de Chacarita. Los otros 26 huetares, se distribuyen entre 10 de los 16 distritos de este cantón.

La ubicación de esta población huetar en distritos urbanos empobrecidos de la vertiente pacífica permite poner en relevancia procesos migratorios más recientes a los estudiados por Amaris (2015) 
que se han dado desde distritos rurales a centros poblacionales dentro de uno o entre varios cantones. Asimismo, en el cantón de Parrita, la población huetar es minúscula, no superando las cinco personas, aunque los indígenas sin pueblo suman 185 individuos de los cuales 103 nacieron en este cantón. Por su parte, en el cantón de Quepos, antes llamado Aguirre, hay 31 huetares en total, sólo en el distrito de Quepos viven 24 huetares; 14 de estos nacieron en este cantón. Los otros 10 huetares están distribuidos en los otros distritos de este cantón. Sin embargo, en dicho cantón se reportan 297 indígenas sin pueblo de los cuales 137 afirman haber nacido en este cantón.

Tabla 6

Indígenas huetares y sin pueblo residentes en cantones puntarenenses (Región Pacífico Central) según lugar de nacimiento (2011)

\begin{tabular}{|l|r|r|r|r|r|}
\hline \multicolumn{1}{|c|}{ Cantones } & Nacidos en este cantón & Nacidos en otro cantón & Sin Pueblo nacido en este cantón & Sin pueblo nacidos en otro cantón \\
\hline Puntarenas & 30 & 18 & 367 & \\
\hline Esparza & 4 & 4 & 86 & 36 \\
\hline Montes de Oro & & 1 & 143 \\
\hline Aguirre (Quepos) & 20 & 11 & 137 & 103 \\
\hline Parrita & 5 & 7 & 36 & \\
\hline Garabito & 1 & 4 & 52 \\
\hline Total & 60 & 45 & 51 \\
\hline
\end{tabular}

Fuente: Elaboración propia con datos del INEC (2011)

Al igual que en el análisis realizado en los cantones de San José, si bien no es posible afirmar que en el Pacífico Central la población indígena sin pueblo sea huetar, es muy probable que al menos las personas que se reconocen como indígenas sin pueblo y hayan nacido en los cantones de Quepos y Parrita, aunque hayan olvidado o siquiera considerado su pertinencia a un pueblo particular, sí lo sean. Lo anterior partiendo de entrevistas realizadas entre el 2012 y el 2015 donde queda claro que más que la identificación huetar muchos mayores y sus familias resaltan su vivencia y tradiciones como indígenas simplemente y sin adscripción a un pueblo específico. Es válido recordar que los nombres con los que se identifica a los pueblos indígenas fueron dados por los españoles durante la conquista y finalmente, en muchos casos, adoptados por estos grupos.

\section{Una mirada a los grandes grupos de ocupación de las poblaciones que se consideran a sí mismas como indígena huetar, indígena sin pueblo y como "blancos o mestizos" registradas en el Censo del 2011}

Es pertinente señalar que entre los conjuntos de ocupaciones que realiza el INEC, se incluyen actividades muy diversas que impiden un análisis más concluyente. Por ejemplo, en ocupaciones elementales, se incluyen tanto peones agrícolas como peones de la construcción. Sin embargo, es oportuno conocer esta clasificación para el análisis que se expone en este artículo. A continuación se cita en detalle la descripción ofrecida por el INEC (2011b: 22-24):

\section{Directores y gerentes}

Comprende las ocupaciones cuyas tareas principales son planificar, dirigir y coordinar la actividad general de las empresas, gobiernos y otras organizaciones y de los departamentos de los mismos, así como formular y revisar la estrategia de las empresas, así también las leyes y reglamentos de los gobiernos.

\section{Profesionales científicos e intelectuales}

Comprende las ocupaciones cuyas tareas principales requieren para su desempeño conocimientos profesionales de alto nivel y experiencia en materia de ciencias físicas y biológicas o ciencias sociales, 
humanidades y artísticas. Sus tareas consisten en desarrollar y aplicar el acervo de conocimientos científicos e intelectuales a los diferentes campos o, por medio de la enseñanza, asegurar la difusión sistemática de estos conocimientos.

\section{Técnicos y profesionales de nivel medio}

Comprende las ocupaciones cuyas tareas principales requieren para su desempeño conocimientos de carácter técnico y la experiencia necesaria para servir de apoyo en labores de carácter administrativo con cierto grado de responsabilidad.

\section{Personal de apoyo administrativo}

Comprende las ocupaciones cuyas tareas requieren los conocimientos y la experiencia necesaria para ordenar, almacenar, procesar y encontrar información. Las tareas consisten en realizar trabajos de secretaría relacionados con la contabilidad, la producción, el almacenamiento y transporte, reservas de viajes, servicio de correos y recepción.

\section{Trabajadores de los servicios y vendedores de comercios y mercados}

Comprende las ocupaciones en el que el desempeño de las tareas principales requiere los conocimientos y la experiencia necesarios para la prestación de diversos servicios y cuidados personales, servicios de protección y de seguridad o la venta de mercancías. Dichas tareas consisten en servicios relacionados con los cuidados personales, la protección de personas y bienes, el mantenimiento del orden público o la venta de mercancías en un comercio o en los mercados.

\section{Agricultores y trabajadores calificados agropecuarios, forestales y pesqueros}

Comprende las ocupaciones en el que el desempeño de las tareas principales requiere los conocimientos y la experiencia necesaria para la obtención de productos de la agricultura, la ganadería, la silvicultura y la pesca. Sus tareas consisten en practicar la agricultura a fin de obtener sus productos, criar o cazar animales, pescar o criar peces y conservar y explotar los bosques.

\section{Oficiales, operarios y artesanos de artes mecánicas y de otros oficios}

Comprende las ocupaciones cuyas tareas principales requieren los conocimientos y la experiencia necesarios para ejercer oficios y profesiones de tipo tradicional en la industria y la construcción, en los que lo esencial es el conocimiento de la materia prima utilizada, de las etapas en el proceso de producción o, de la naturaleza y las aplicaciones de los productos fabricados. Evidentemente, en la realización de las áreas anteriores se puede utilizar maquinaria avanzada tecnológicamente sin que ello suponga un cambio en la cualificación básica y en los conocimientos requeridos.

\section{Operadores de instalaciones y máquinas y ensambladores}

Comprende las ocupaciones cuyas tareas requiere los conocimientos y la experiencia necesarios para atender y vigilar el funcionamiento de máquinas e instalaciones industriales de gran tamaño, y a menudo automatizadas, que reducen el esfuerzo físico y el tiempo requerido en la realización del trabajo. Las tareas y deberes de estas ocupaciones precisan de un conocimiento para lograr que las máquinas rindan óptimamente. Estas ocupaciones están orientadas al conocimiento y manejo de las máquinas siendo menos importante el conocimiento de la materia prima, los procesos de transformación y sus resultados. 


\section{Ocupaciones elementales}

Comprende las ocupaciones cuyas tareas requieren los conocimientos y la experiencia necesaria para realizar tareas, generalmente, sencillas y rutinarias, realizadas con la ayuda de herramientas manuales, y para las cuales se precisa a veces de un esfuerzo físico considerable y, salvo raras excepciones, escasa iniciativa. Sus tareas consisten en ejecutar tareas simples relacionadas con la minería, la agricultura o la pesca, la industria manufacturera y la construcción, así como realizar labores de limpieza y asistencia doméstica o el reparto de publicidad o prensa en la calle.

Ahora bien, si se analizan los conjuntos de las principales ocupaciones (ocupación gran grupo) ordenados por el INEC (2011b) a nivel nacional para personas que consideran indígenas en general, indígenas huetares, indígenas sin pueblo y para los "blancos o mestizos", obtenemos que los huetares así como los indígenas sin pueblo comparten en las mismas proporciones de ocupación labores similares salvo aquellas reunidas en el gran grupo de Ocupaciones elementales donde $29 \%$ de los huetares se dedican a estas mientras que $25 \%$ de los indígenas sin pueblo obtienen sus ingresos de las mismas. Otra excepción que vale la pena adicionar cuando se contrastan estos dos grupos es que $10 \%$ de las personas autoidentificadas como indígenas son Técnicos y profesionales de nivel medio entretanto que los huetares $7 \%$.

Pareciera, asimismo, desprenderse de la Tabla 7 que los porcentajes de población dedicada a algunos de los grandes conjuntos ocupaciones agrupados por el INEC difieren si son ejecutadas por huetares o si las mismas son realizadas por personas que se consideran a sí mismas indígenas en general. En este sentido, se observa como $11 \%$ de los indígenas huetares se dedican a actividades congregadas dentro del gran grupo de Profesionales, científicos e intelectuales mientras que solo $9 \%$ de los indígenas, en general, se dedican a empleos reunidos dentro de este grupo ocupacional. Por otra parte, $21 \%$ de los huetares se emplea como Trabajadores de los servicios y vendedores en comercios y mercados mientras que $17 \%$ de los indígenas trabaja en las actividades allí reunidas. Esto podría deberse al peso que tienen, por ejemplo, cabécares y bribris más dedicados a la agricultura y lejos de centros poblacionales importantes. Nótese igualmente (Tabla 7) el mayor porcentaje de población que se autoidentifica como indígena que cuenta con oficios dentro del grupo de Trabajadores agricultores, agropecuarios calificados y forestales alcanzando $15 \%$ mientras que en los huetares solo 7\%. Esto podría obedecer además de la presión sobre la tierra, ya anotada anteriormente, al hecho de que el territorio indígena de Quitirrisí está a una hora de la ciudad capital así también de otros centros urbanos del Gran Área Metropolitana (GAM) lo que les lleva a los miembros de este pueblo a insertarse también en actividades relacionadas con el gran grupo Operarios de instalaciones, máquinas y ensambladoras donde los huetares dedicados a este tipo de labores alcanzan el 7\% mientras que el resto de indígenas 5\%. La otra diferencia, nada desdeñable, se revela en la columna de las Ocupaciones elementales que en el caso de los indígenas ocupa al $33 \%$ mientras que en los huetares $29 \%$.

Tabla 7

Porcentajes por grandes grupos de ocupación según indígenas huetares e indígenas sin pueblo, población que se considera a sí misma como indígena y

"blancos o mestizos" (Costa Rica, 2011)

\begin{tabular}{|c|c|c|c|c|c|c|c|c|c|c|}
\hline & $\begin{array}{l}\text { Nivel direct } \\
\text { adm público y } \\
\text { empr privada }\end{array}$ & $\begin{array}{c}\text { Nivel } \\
\text { profesio, } \\
\text { cientif e } \\
\text { intelect }\end{array}$ & \begin{tabular}{|c|} 
Nivel \\
técnico y \\
profesional \\
medio
\end{tabular} & $\begin{array}{c}\text { Apoyo } \\
\text { administrat } \\
\text { ivo }\end{array}$ & $\begin{array}{c}\text { Venta } \\
\text { locales y } \\
\text { prestac } \\
\text { serv direct }\end{array}$ & $\begin{array}{l}\text { Agropecua } \\
\text { rias, } \\
\text { agrícolas y } \\
\text { pesqueras } \\
\text { calificadas }\end{array}$ & $\begin{array}{c}\text { Producción } \\
\text { artesan, const, } \\
\text { mecan, art graf } \\
\text { y manuf } \\
\text { calificadas }\end{array}$ & $\begin{array}{l}\text { Operación de } \\
\text { instalaciones, } \\
\text { máquinas y } \\
\text { ensambladores }\end{array}$ & $\begin{array}{l}\text { Ocupaciones } \\
\text { elementales }\end{array}$ & Total \\
\hline Huetares & 1 & 11 & 7 & 5 & 21 & 7 & 12 & 7 & 29 & 100 \\
\hline Sin Pueblo & 1 & 11 & 10 & 6 & 21 & 6 & 12 & 8 & 25 & 100 \\
\hline Sí se considera indígena & 1 & 9 & 6 & 4 & 17 & 15 & 10 & 5 & 33 & 100 \\
\hline No se considera indígena & 2 & 14 & 10 & 8 & 20 & 4 & 12 & 9 & 21 & 100 \\
\hline
\end{tabular}


Ahora bien, al incluir en este ejercicio comparativo a nivel nacional aquellas actividades en las cuales laboran los huetares frente a las realizadas por la población "blanca o mestiza", se encuentra que esta última supera a la otra en cuanto a la proporción de sus individuos en actividades mejor calificadas y, además, que los "blancos o mestizos" proporcionalmente se dedican con menos frecuencia a los grandes grupos que requieren menor calificación y por las que se obtienen menores ingresos. Lo anterior, apoyado en los datos expuestos, motiva a indagar sobre una posible segregación socio-laboral. Esto es relevante todavía más cuando se examinan y se contrastan los porcentajes entre los "blancos o mestizos" y los indígenas sin pueblo y los indígenas en general. Se distingue entonces cómo los "blancos o mestizos" en Costa Rica se dedican en menor proporción a Ocupaciones elementales, un poco menos a las ventas en comercios y servicios, pero un mayor porcentaje de los individuos autoidentificados como "blancos o mestizos" obtiene ingresos de actividades profesionales, científicas e intelectuales. Se destaca, igualmente, una mayor proporción de personas que se autoidentifican como "blancos o mestizos" realizando tareas relacionadas con la dirección y gerencia, además como Personal de apoyo administrativo.

\section{Conclusiones}

Si se consideran los fenómenos expulsivos en el cantón de Mora y Puriscal para la primera mitad del siglo XX descritos por Amaris (2015) se puede concluir que los huetares continúan experimentando un proceso de desterritorialización que consiste básicamente en la pérdida de control cultural que merma la capacidad de decisión sobre los componentes territoriales necesarios para solventar insuficiencias y resolver sus problemas (Bonfil, 1988). Ello se origina en la desventajosa tenencia de la tierra en sus territorios indígenas, ya comentada, pero también en el estímulo creciente, dada la cercanía, a desplazarse a los centros urbanos de la GAM donde, mayoritariamente, encuentran empleo en las ocupaciones relacionadas con los servicios y oficios de carácter urbano semi-industrial. Si bien es cierto, la situación ocupacional de las personas autoidentificadas como huetares, según se anota del Censo del 2011, pareciera ser apenas más favorable que los indígenas en general, una gran parte de sus miembros se dedica a labores poco remuneradas que requieren escasa calificación. Lo precedente, a juzgar por la frágil economía doméstica explicada durante las entrevistas realizadas por Amaris (2015), estuvo relacionada en el pasado con dificultades para alcanzar el sustento debido a la privatización de tierras de uso común, fuente de alimentos y leña, así como la pérdida de fertilidad de la tierra por la intensificación de los cultivos teniendo que complementar actividades económicas. Lo que este pueblo experimenta en el presente, por lo tanto, pareciera ser la continuidad de una lógica de despojo que apenas se logra mitigar debido al papel que tiene la existencia de estos dos territorios (Quitirrisí y Zapatón) para este pueblo.

Es necesario reiterar que, del examen realizado al comenzar este escrito, el $68 \%$ de la población del pueblo huetar se concentra en la provincia de San José y que, de estos, el 58\% habita en los territorios indígenas de Quitirrisí y Zapatón siendo, entonces, la provincia de San José la que reúne al mayor número de miembros de este pueblo. Esto permite concluir que la reciente constitución de los territorios indígenas de Quitirrisí y Zapatón por parte del Estado costarricense continúa siendo entonces, pese a todo, un aliciente para albergar a la población indígena huetar en dichos territorios. Sin embargo, dicho estímulo resulta insuficiente a la luz de las problemáticas ya descritas en la primera parte de esta investigación, relacionadas con el despojo y el desestimulo de la agricultura familiar y la pérdida de fertilidad de los suelos. Por otra parte, la proximidad de la ciudad de San José, para el caso del Territorio Indígena de Quitirrisí, incentiva que un gran número de huetares se desplace a ofrecer su fuerza de trabajo a los centros urbanos más poblados de la provincia de San José logrando luego regresar a sus hogares. Igualmente, las ocupaciones a las cuales se dedican los huetares en la provincia de San José, así como la dispersión de sus lugares de residencia entre los distritos del cantón central de San José permiten sostener también que buena parte de los huetares son atraídos para trabajar en los centros urbanos en actividades que no son agropecuarias. 
Los señalamientos anteriores ganan preponderancia, para el caso del pueblo huetar, cuando se observa que su situación de marginalidad en términos nacionales es compartida casi con idéntico carácter a las condiciones de las personas que se autoidentifican como indígenas sin pueblo. Relacionado con este punto es pertinente llamar la atención acerca de la numerosa presencia de personas que se consideran a sí mismas como indígenas sin pueblo en cantones próximos a los territorios indígenas siendo llamativa la situación de Acosta ya que sin contar con territorio indígena un número importante de acosteños se sienten indígenas aunque de ningún pueblo. Lo mismo en Parrita y Quepos. Esta situación se invierte en caso de Mora y Puriscal como ya se vio. Lo anterior sin duda tiene que ver con el establecimiento en las décadas del setenta y ochenta de territorios indígenas en ambos cantones lo cual se convierte en un referente étnico-institucional importante para que aquellos autoidentificados como indígenas se sientan parte de un pueblo como en este caso el huetar.

Es finalmente necesario problematizar acerca de la necesidad de que se separe la categoría de blanco con respecto a la de mestizo en los futuros censos costarricenses, ya que permitiría a la persona entrevistada optar por una mayor especificidad dándole la oportunidad para que exprese con claridad si se considera o no portador del legado cultural histórico precolombino ya que mestizo, a diferencia de blanco, alude al reconocimiento de antecedentes prehispánicos que conforman su identidad junto al legado europeo por parte de al menos uno de sus progenitores o un ancestro. Lo anterior en vista de que la categoría "blanco o mestizo", según el Manual para Censistas se define como: "personas que se identifican principalmente con el legado cultural e histórico hispanoamericano. Se incluye también la identificación con el legado cultural e histórico europeo o anglosajón" (INEC, 2011c: 144). De esta manera se relacionan ambas categorías con el legado europeo o anglosajón lo que resulta confuso e imposibilita incluir a los mestizos en comparaciones más finas como las que se propuso en este estudio.

Para finalizar resulta, entonces, importante señalar que a la luz del análisis elaborado en el presente artículo académico, el número de miembros del pueblo huetar podría ser mayor que el que se autoidentificó expresamente como tal en el censo (INEC, 2011). Lo anterior, si se le suman las personas autoidentificadas como indígenas sin pueblo nacidas en cantones con tradición histórica huetar o bien cantones receptores de migraciones de familias de este pueblo a finales del siglo XIX, primera mitad del siglo XX, pero también migraciones más recientes hacia las provincias de Alajuela y Limón. Esto justifica la necesidad de realizar estudios de carácter cualitativo que complementen y profundicen la información recogida en el censo.

\section{Bibliografía}

Amaris, Orlando. (2015). La economía-mundo y la migración indígena huetar en el cantón de Mora, Costa Rica, 1900 - 1955. Tesis para optar por el grado de Maestría en Geografía, Universidad de Costa Rica.

Bartels, Jorge y Martínez, Ricardo. (2008). El desarrollo de la agricultura en la Región Pacífica Central de Costa Rica. Revista Diálogos. Número especial.

Bartels, Jorge y Martínez, Ricardo. (2010). El desarrollo de la agricultura en la Región Pacífica Central de Costa Rica (1909-1955). En Abarca, Orestes, Bartels, Jorge. y Marín, Juan José. De puerto a región: El Pacífico Central y Sur de Costa Rica (1821-2007). San José: Alam Mater.

Barth, Fredrik. (1976). Introducción. En: Barth, Fredrik (Comp.), Los grupos étnicos y sus fronteras. México: Fondo de Cultura Económica.

Bonfil, Guillermo. (1988).Teoría del control cultural en el estudio de procesos étnicos. Anuario antropológico 86

Consultado en octubre del 2014. Disponible en http://ciesas.edu.mx/Publicaciones/Clasicos/articulos/ TeoriadelControl.pdf 
Botey, Ana María. (1999). El ferrocarril al pacífico: un ente de regulación y desarrollo en crisis permanente (1880-1972). Anuario de Estudios centroamericanos. Vol 25(1). Consultado en diciembre de 2014. Disponible en: http://revistas.ucr.ac.cr/index.php/anuario/article/viewFile/1936/1900

Borge, Carlos. (2007). Consulta en los territorios indígenas del Pacífico de Costa Rica. Regularización de los derechos relacionados con la propiedad inmueble en áreas bajo regímenes especiales (ABRE). San José: Unidad Ejecutora de Regularización del Catastro y Registro.

Defensoría de los Habitantes. (2010). Informe anual de labores. 2009-2010. San José: Autor.

Defensoría de los Habitantes (2014). Informe de labores. 2013-2014. Consultado en febrero del 2015. Disponible en: http://www.asamblea.go.cr/Centro_de_informacion/Secretaria_Directorio/Informe $\% 20$ anual $\% 2020112012 \% 20 \mathrm{de} \% 201 \mathrm{a} \% 20$ Defensora $\% 20 \mathrm{de} \% 201 \mathrm{os} \% 20 \mathrm{Habi} /$ Informe\%20anual\%202013-2014\%20de\%201a\%20Defensor\%C3\%ADa\%20de\%20los\%20Habitantes/ Informe\%20de\%20labores\%202013-2014_al.pdf

INEC. (2011). X Censo nacional de población. Características sociales. Consultado en abril del 2015. Disponible en http://www.inec.go.cr/Web/Home/GeneradorPagina.aspx

INEC. (2011a). Territorios indígenas. Principales indicadores demográficos y socioeconómicos. Consultado en abril del 2015. Disponible en http://www.uned.ac.cr/extension/images/ifcmdl/02 . Censo_2011._Territorios_Indigenas.pdf

INEC. (2011b). Clasificación de ocupaciones de Costa Rica. Consultado de febrero del 2016. Disponible en http://www.inec.go.cr/A/MI/Publicaciones/Serie\%20Metodol\%C3\%B3gica/ Metodolog\%C3\%ADas/38.\%20Metodolog\%C3\%ADa\%20Ocupaciones\%202011.pdf

INEC. (2011c). Manual para Censistas. Consultado de febrero del 2016. Disponible en http://www.inec. go.cr/A/MS/Censos/Censo\%202011/Censo\%202011/03.\%20Manual\%20para\%20Censista.pdf

Quesada, Miguel Ángel. (1996). Los huetares: historia, lengua, etnografía y tradición oral. Cartago: Editorial Tecnológica de Costa Rica.

Quesada, Miguel Ángel. (1998). Tradiciones huetares. Heredia: Editorial de la Universidad Nacional.

Quesada, Miguel Ángel. (1999-2000). Situación actual y futuro de las lenguas indígenas de Costa Rica. Estudios de lingüística chibcha. Tomos XVIII-XIX. 
